Antoni Leon Dawidowicz (Kraków)

Anna Poskrobko (Białystok)

JERZY LESZEK ZALASIŃSKi (Tarnów)

\title{
ON THE AGE-DEPENDENT PREDATOR-PREY MODEL
}

Abstract. The paper deals with the description of a model which is the synthesis of two classical models, the Lotka-Volterra and McKendrick-von Foerster models. The existence and uniqueness of the solution for the new population problem are proved, as well the asymptotic periodicity but under some simplifying assumptions.

1. Introduction. The best known model in the classical mathematical ecology is the Lotka-Volterra model [13]. It describes the competition of two populations, predators and preys. This model is based on a system of ordinary differential equations and leads to periodic solutions. The classical Lotka-Volterra model assumes that each contact of a predator with a prey finishes with the predator eating the prey. But it is a certain idealization. In fact such a contact is the beginning of a fight (e.g. chase), the result of which depends on features of both sides, particularly, their age. Moreover, the "chance" for such a contact depends also on environmental conditions. So a natural modification of the model takes age structure into consideration. Such an age-dependent model, but for a single population, comes from McKendrick [12] and von Foerster [14]. There are many other modifications of the classical Lotka-Volterra model dealing with the dynamics of preypredator systems, for example a prey-predator system in specific habitat with two zones, free and reserved [9]; multi-dimensional Lotka-Volterra system [10] or Lotka-Volterra multi-species systems [1, 2]. Other papers analyse age-dependent prey-predator systems [5] but with delays [4, [15] or diffusion [8]. However in these papers the problem of dependence on the age is

2010 Mathematics Subject Classification: Primary 35F50; Secondary 92D25, 35B10, 35R09. Key words and phrases: population model, Lotka-Volterra, age-dependent predator-prey model, existence and uniqueness theorem. 
analyzed only fragmentarily. In this paper we assume that the "chance" that a prey is eaten by a predator depends on the predator's age as well as on the prey's. In Section 2 we introduce the variables of the model and the basic assumptions. We formulate the population problem and define its solution in Section 3. Constructing the solution consists in applying the method of characteristics for an auxiliary problem with a classical boundary condition and on describing this condition as a fixed-point for an appropriate transformation. Section 4 contains the proof that the transformation is contractive in the space of continuous functions with a Bielecki-type norm. In Section 5 we compare the classical Lotka-Volterra model with ours. It is shown that if the coefficients of the model are independent of the age then the global biomass satisfies the classical Lotka-Volterra equation, and is a periodic function of time. In this situation the age structure is also asymptotically periodic. This fact is shown in the last section.

2. Variables, parameters and assumptions of the model. There are two basic variables in our model:

- $u_{1}(t, x)$ denotes the density of the population of predators of age $x$ at time $t$,

- $u_{2}(t, x)$ denotes the density of the population of preys of age $x$ at time $t$.

We assume that predators generally die a natural death. We can express their mortality by the classical McKendrick-von Foerster equation

$$
\frac{\partial u_{1}}{\partial t}+\frac{\partial u_{1}}{\partial x}=-\lambda(x) u_{1}(t, x) .
$$

Preys die being eaten by a predator. We assume that the parameter $\alpha(x, y)$ denotes the "chance" that a prey of age $x$ is eaten by a predator of age $y$. Then the equation describing the mortality of preys has the form

$$
\frac{\partial u_{2}}{\partial t}+\frac{\partial u_{2}}{\partial x}=-\int_{0}^{\infty} \alpha(x, y) u_{1}(t, y) d y \cdot u_{2}(t, x) .
$$

It remains to introduce the "renewal" equations. For a prey it is of the form

$$
u_{2}(t, 0)=\int_{0}^{\infty} \beta(x) u_{2}(t, x) d x .
$$

For predators, we should consider in such an equation a "transformation" of eaten prey into offspring of predators. Therefore

$$
u_{1}(t, 0)=k \int_{0}^{\infty} \int_{0}^{\infty} \alpha(x, y) u_{2}(t, x) u_{1}(t, y) d x d y \text {. }
$$


3. Formulation of the problem. We consider the system (2.1)-2.4 with the initial conditions

$$
u_{1}(0, x)=v_{1}(x), \quad u_{2}(0, x)=v_{2}(x)
$$

where $v_{1}, v_{2}$ are continuous non-negative and integrable functions $[0, \infty) \rightarrow \mathbb{R}$ satisfying the conditions

$$
v_{1}(0)=k \int_{0}^{\infty} \int_{0}^{\infty} \alpha(x, y) v_{2}(x) v_{1}(y) d x d y, \quad v_{2}(0)=\int_{0}^{\infty} \beta(x) v_{2}(x) d x .
$$

Let $\varphi=\left(\varphi_{1}, \varphi_{2}\right):[0, T] \rightarrow \mathbb{R}^{2}$, where $T>0$, be a continuous function satisfying the conditions

$$
\varphi_{1}(0)=v_{1}(0), \quad \varphi_{2}(0)=v_{2}(0) .
$$

We first consider an auxiliary problem: equations $(2.1), 2.2$ with the conditions (3.1), (3.2) and

$$
u_{1}(t, 0)=\varphi_{1}(t), \quad u_{2}(t, 0)=\varphi_{2}(t) .
$$

A solution of this problem is

$$
u_{1}(t, x)= \begin{cases}\varphi_{1}(t-x) e^{-\int_{0}^{x} \lambda(s) d s} & \text { for } x \leq t \\ v_{1}(x-t) e^{-\int_{0}^{t} \lambda(x-s) d s} & \text { for } x>t\end{cases}
$$

and

$$
u_{2}(t, x)= \begin{cases}\varphi_{2}(t-x) e^{-\int_{t-x}^{t} R(s, x+s-t) d s} & \text { for } x \leq t, \\ v_{2}(x-t) e^{-\int_{0}^{t} R(t-s, x-s) d s} & \text { for } x>t\end{cases}
$$

where

$$
R(t, x)=\int_{0}^{\infty} \alpha(x, y) u_{1}(t, y) d y .
$$

The above solution can be obtained in the following way (see [11 or [6]). Let $\left(t_{0}, x_{0}\right) \in[0, T] \times \mathbb{R}_{+}$and let $\bar{u}_{1}(h)=u_{1}\left(t_{0}+h, x_{0}+h\right), \bar{u}_{2}(h)=$ $u_{2}\left(t_{0}+h, x_{0}+h\right), \bar{\lambda}(h)=\lambda\left(x_{0}+h\right)$ and $\bar{R}(h)=R\left(t_{0}+h, x_{0}+h\right)$. Then (2.1) and (2.2) imply

$$
\frac{d \bar{u}_{1}}{d h}+\bar{\lambda}(h) \bar{u}_{1}=0 \quad \text { and } \quad \frac{d \bar{u}_{2}}{d h}+\bar{R}(h) \bar{u}_{2}=0 .
$$

These equations have unique solutions, respectively

$$
u_{1}\left(t_{0}+h, x_{0}+h\right)=u_{1}\left(t_{0}, x_{0}\right) e^{-\int_{0}^{h} \lambda(x+s) d s}
$$

and

$$
u_{2}\left(t_{0}+h, x_{0}+h\right)=u_{2}\left(t_{0}, x_{0}\right) e^{-\int_{0}^{h} R\left(t_{0}+s, x_{0}+s\right) d s},
$$

giving the values of $u_{1}$ and $u_{2}$ at all points on the characteristics through $\left(t_{0}, x_{0}\right)$. In particular, if we take for $x \leq t,\left(t_{0}, x_{0}\right)=(t-x, 0)$ and $h=x$ in 
(3.6) and (3.7) we get

$$
\begin{aligned}
& u_{1}(t, x)=\varphi_{1}(t-x) e^{-\int_{0}^{x} \lambda(s) d s}, \\
& u_{2}(t, x)=\varphi_{2}(t-x) e^{-\int_{t-x}^{t} R(s, x+s-t) d s} .
\end{aligned}
$$

On the other hand, writing $\left(t_{0}, x_{0}\right)=(0, x-t)$ and $h=t$ in (3.6) and (3.7) we get for $x \leq t$ the following solutions:

$$
\begin{aligned}
& u_{1}(t, x)=v_{1}(x-t) e^{-\int_{0}^{t} \lambda(x-s) d s}, \\
& u_{2}(t, x)=v_{2}(x-t) e^{-\int_{0}^{t} R(t-s, x-s) d s} .
\end{aligned}
$$

Define the operator $\Theta: C\left([0, T], \mathbb{R}^{2}\right) \rightarrow C\left([0, T], \mathbb{R}^{2}\right)$,

$$
\Theta \varphi=\left((\Theta \varphi)_{1},(\Theta \varphi)_{2}\right):[0, T] \rightarrow \mathbb{R}^{2},
$$

by the formula

$$
(\Theta \varphi)_{1}(t)=k \int_{0}^{\infty} R(t, x) u_{2}(t, x) d x, \quad(\Theta \varphi)_{2}(t)=\int_{0}^{\infty} \beta(x) u_{2}(t, x) d x .
$$

Clearly, the space $C\left([0, T], \mathbb{R}^{2}\right)$ with the usual norm

$$
\|\varphi\|=\sup _{t \in[0, T]}\left(\left|\varphi_{1}(t)\right|+\left|\varphi_{2}(t)\right|\right)
$$

is a Banach space.

Now we shall define solutions of the system (2.1)-(3.1) for $t \in[0, T]$, where $T>0$.

Definition 3.1. A solution to (2.1)-2.4 with the initial conditions (3.1) is the function $u=\left(u_{1}, u_{2}\right) \in L^{1}\left([0, T] \times \mathbb{R}_{+}, \mathbb{R}^{2}\right)$ defined by (3.4), (3.5), when the function $\varphi$ is a fixed point of the operator $\Theta$, i.e. $\Theta \varphi=\varphi$.

REMARK. A classical solution to (2.1) $-(2.4)$ with the initial conditions (3.1) is a solution in the sense of Definition 3.1.

\section{Main theorem}

Main Theorem 4.1. Let $\alpha, \beta, \lambda \geq 0$. Assume also that $\beta \in L^{\infty}(0, \infty)$ and $\alpha \in L^{\infty}\left((0, \infty)^{2}\right)$. Then the system of differential equations 2.1)-2.4 with the initial conditions (3.1) has exactly one non-negative solution on the set

$$
x \geq 0, \quad t \in[0, T] .
$$

Proof. Let $\mathcal{X}_{T}$ denote the space of all continuous, non-negative functions $\varphi:[0, T] \rightarrow \mathbb{R}^{2}$ satisfying the conditions 3.2 and the estimate

$$
\varphi_{2}(t) \leq \bar{\beta} e^{\bar{\beta} t}\left\|v_{2}\right\|_{L^{1}} \quad \text { where } \quad \bar{\beta}=\|\beta\|_{L^{\infty}} .
$$

To prove that $\Theta: \mathcal{X}_{T} \rightarrow \mathcal{X}_{T}$, we first show that $\Theta \varphi:[0, T] \rightarrow \mathbb{R}^{2}$ is continuous. 
We claim that the families $\left\{u_{i}(t, \cdot)\right\}_{t \in[0, T]}$ are uniformly summable. Select some $\varepsilon>0$. From the summability of $v_{i}$ there exists $c>0$ such that

$$
\int_{\left|v_{i}(x)\right|>c}\left|v_{i}(x)\right| d x<\varepsilon .
$$

Let $c^{\prime}=\max \left\{c, \max _{t \in[0, T]} \varphi_{i}(t)\right\}$. From (3.4), 3.5) it follows that

$$
\left\{x: u_{i}(t, x)>c^{\prime}\right\} \subset\left\{x: v_{i}(x-t)>c\right\},
$$

which proves the claim.

Since $\alpha$ does not depend on $t$, the boundedness of $\alpha$ yields the uniform boundedness of $R$ with respect to $t$. Consequently the families

$$
\left\{R(t, \cdot) u_{2}(t, \cdot): t \in[0, T]\right\} \text { and }\left\{\beta(\cdot) u_{2}(t, \cdot): t \in[0, T]\right\}
$$

are uniformly summable. From this we conclude that the integrals

$$
\int_{0}^{\infty} R(t, x) u_{2}(t, x) d x \text { and } \int_{0}^{\infty} \beta(x) u_{2}(t, x) d x
$$

are continuous with respect to $t$. Since

$$
(\Theta \varphi)_{1}(t)=k \int_{0}^{\infty} R(t, x) u_{2}(t, x) d x \quad \text { and } \quad(\Theta \varphi)_{2}(t)=\int_{0}^{\infty} \beta(x) u_{2}(t, x) d x,
$$

the functions $(\Theta \varphi)_{1}$ and $(\Theta \varphi)_{2}$ are continuous.

To prove that the function $(\Theta \varphi)_{2}$ satisfies the inequality (4.1) we estimate

$$
\begin{aligned}
(\Theta \varphi)_{2}(t)= & \int_{0}^{\infty} \beta(x) u_{2}(t, x) d x \\
= & \int_{0}^{t} \beta(x) \varphi_{2}(t-x) e^{-\int_{t-x}^{t} R(s, x+s-t) d s} d x \\
& +\int_{t}^{\infty} \beta(x) v_{2}(x-t) e^{-\int_{0}^{t} R(t-s, x-s) d s} d x \\
\leq & \int_{0}^{t} \beta(x) \varphi_{2}(t-x) d x+\int_{t}^{\infty} \beta(x) v_{2}(x-t) d x \\
= & \int_{0}^{t} \beta(t-x) \varphi_{2}(x) d x+\int_{0}^{\infty} \beta(x+t) v_{2}(x) d x \\
\leq & \bar{\beta} \int_{0}^{t} \bar{\beta} e^{\bar{\beta} x} d x\left\|v_{2}\right\|_{L^{1}}+\bar{\beta}\left\|v_{2}\right\|_{L^{1}}=\bar{\beta} e^{\bar{\beta} t}\left\|v_{2}\right\|_{L^{1}} .
\end{aligned}
$$

This means that $\Theta: \mathcal{X}_{T} \rightarrow \mathcal{X}_{T}$. 
Let now $\varphi, \bar{\varphi} \in \mathcal{X}_{T}$. Let $\bar{u}_{1}, \bar{u}_{2}$ be given by the formulas (3.4), 3.5 with $\varphi$ replaced by $\bar{\varphi}$ and let

$$
\bar{R}(t, x)=\int_{0}^{\infty} \alpha(x, y) \bar{u}_{1}(t, y) d y .
$$

We shall estimate the differences $\left|u_{i}(t, x)-\bar{u}_{i}(t, x)\right|$ for $i=1,2$. For $x \leq t$ we have

$$
\begin{aligned}
\left|u_{1}(t, x)-\bar{u}_{1}(t, x)\right| & =\left|\varphi_{1}(t-x) e^{-\int_{0}^{x} \lambda(s) d s}-\bar{\varphi}_{1}(t-x) e^{-\int_{0}^{x} \lambda(s) d s}\right| \\
& \leq\left|\varphi_{1}(t-x)-\bar{\varphi}_{1}(t-x)\right| .
\end{aligned}
$$

For $x>t$ clearly $u_{1}(t, x)=\bar{u}_{1}(t, x)$. Hence

$$
\begin{aligned}
|R(t, x)-\bar{R}(t, x)| & \leq \int_{0}^{t} \alpha(x, y)\left|\varphi_{1}(t-y)-\bar{\varphi}_{1}(t-y)\right| d y \\
& \leq \sup _{y \in[0, t]} \alpha(x, y) \int_{0}^{t}\left|\varphi_{1}(s)-\bar{\varphi}_{1}(s)\right| d s \\
& \leq \alpha_{0}(x) \int_{0}^{t}\left|\varphi_{1}(y)-\bar{\varphi}_{1}(y)\right| d y
\end{aligned}
$$

where $\alpha_{0}(x)=\sup _{y \in[0, t]} \alpha(x, y)$. From the above it follows that

$$
\begin{aligned}
& \left|e^{-\int_{t-x}^{t} R(s, x+s-t)} d s-e^{-\int_{t-x}^{t} \bar{R}(s, x+s-t) d s}\right| \\
& \leq\left|\int_{t-x}^{t} R(s, x+s-t) d s-\int_{t-x}^{t} \bar{R}(s, x+s-t) d s\right| \\
& \leq \int_{t-x}^{t} \alpha_{0}(x+s-t) \int_{0}^{s}\left|\varphi_{1}(y)-\bar{\varphi}_{1}(y)\right| d y d s \\
& \leq \int_{0}^{x} \alpha_{0}(x-s) \int_{0}^{t-s}\left|\varphi_{1}(y)-\bar{\varphi}_{1}(y)\right| d y d s \\
& \leq \int_{0}^{t}\left|\varphi_{1}(y)-\bar{\varphi}_{1}(y)\right| \int_{0}^{t-y} \alpha_{0}(x-s) d s d y \\
& \leq t \sup _{z \in[0, t]} \alpha_{0}(z) \int_{0}^{t}\left|\varphi_{1}(y)-\bar{\varphi}_{1}(y)\right| d y .
\end{aligned}
$$

We now estimate the difference $\left|u_{2}(t, x)-\bar{u}_{2}(t, x)\right|$. For $x>t$,

$$
\begin{aligned}
\left|u_{2}(t, x)-\bar{u}_{2}(t, x)\right| & =v_{2}(x-t)\left|e^{-\int_{0}^{t} R(t-s, x-s) d s}-e^{-\int_{0}^{t} \bar{R}(t-s, x-s) d s}\right| \\
& \leq v_{2}(x-t) \int_{0}^{t}|R(t-s, x-s)-\bar{R}(t-s, x-s)| d s
\end{aligned}
$$




$$
\begin{aligned}
& =v_{2}(x-t) \int_{0}^{t}|R(s, x-t+s)-\bar{R}(s, x-t+s)| d s \\
& \leq v_{2}(x-t) \int_{0}^{t} \alpha_{0}(x-t+s) \int_{0}^{s}\left|\varphi_{1}(y)-\bar{\varphi}_{1}(y)\right| d y d s \\
& \leq v_{2}(x-t) \int_{0}^{t} \alpha_{0}(x-s) \int_{0}^{t-s}\left|\varphi_{1}(y)-\bar{\varphi}_{1}(y)\right| d y d s \\
& =v_{2}(x-t) \int_{0}^{t}\left|\varphi_{1}(y)-\bar{\varphi}_{1}(y)\right| \int_{0}^{t-y} \alpha_{0}(x-s) d s d y \\
& \leq t v_{2}(x-t) \cdot \sup _{z \in[0, t]} \alpha_{0}(z) \int_{0}^{t}\left|\varphi_{1}(y)-\bar{\varphi}_{1}(y)\right| d y,
\end{aligned}
$$

while for $x \leq t$,

$$
\begin{aligned}
& \left|u_{2}(t, x)-\bar{u}_{2}(t, x)\right| \\
& =\left|\varphi_{2}(t-x) e^{-\int_{t-x}^{x} R(s, x+s-t) d s}-\bar{\varphi}_{2}(t-x) e^{-\int_{t-x}^{x} \bar{R}(s, x+s-t) d s}\right| \\
& \leq\left|\left(\varphi_{2}(t-x)-\bar{\varphi}_{2}(t-x)\right) e^{-\int_{t-x}^{x} R(s, x+s-t) d s}\right| \\
& \quad \quad+\left|\bar{\varphi}_{2}(t-x)\left(e^{-\int_{t-x}^{x} R(s, x+s-t) d s}-e^{-\int_{t-x}^{x} \bar{R}(s, x+s-t) d s}\right)\right| \\
& \leq\left|\varphi_{2}(t-x)-\bar{\varphi}_{2}(t-x)\right|+t \bar{\varphi}_{2}(t-x) \sup _{z \in[0, t]} \alpha_{0}(z) \int_{0}^{t}\left|\varphi_{1}(y)-\bar{\varphi}_{1}(y)\right| d y .
\end{aligned}
$$

Now, we estimate $\left|(\Theta \varphi)_{1}(t)-(\Theta \bar{\varphi})_{1}(t)\right|$ and $\left|(\Theta \varphi)_{2}(t)-(\Theta \bar{\varphi})_{2}(t)\right|$. First,

$$
\begin{gathered}
\left|(\Theta \varphi)_{1}(t)-(\Theta \bar{\varphi})_{1}(t)\right| \leq k \int_{0}^{t}\left|u_{2}(t, x) R(t, x)-\bar{u}_{2}(t, x) \bar{R}(t, x)\right| d x \\
\quad+k \int_{t}^{\infty}\left|u_{2}(t, x) R(t, x)-\bar{u}_{2}(t, x) \bar{R}(t, x)\right| d x \\
\leq k \int_{0}^{t} R(t, x)\left|u_{2}(t, x)-\bar{u}_{2}(t, x)\right| d x+k \int_{0}^{t} \bar{u}_{2}(t, x)|R(t, x)-\bar{R}(t, x)| d x \\
\left.+k \int_{t}^{\infty} u_{2}(t, x)|R(t, x)-\bar{R}(t, x)| d x+k \int_{t}^{\infty} \bar{R}(t, x) \mid u_{2}(t, x)-\bar{u}_{2}(t, x)\right) \mid d x \\
\leq K_{1}(T) \int_{0}^{t}\left(\left|\varphi_{1}(x)-\bar{\varphi}_{1}(x)\right|+\left|\varphi_{2}(x)-\bar{\varphi}_{2}(x)\right|\right) d x
\end{gathered}
$$

for some $K_{1}$ depending only on $T$. The dependence follows from formula 
(3.5), that is, summability of $u_{2}$ and $v_{2}$. Analogously

$$
\left|(\Theta \varphi)_{2}(t)-(\Theta \bar{\varphi})_{2}(t)\right| \leq K_{2}(T) \int_{0}^{t}\left(\left|\varphi_{1}(x)-\bar{\varphi}_{1}(x)\right|+\left|\varphi_{2}(x)-\bar{\varphi}_{2}(x)\right|\right) d x
$$

and so

$$
\begin{aligned}
\left|(\Theta \varphi)_{1}(t)-(\Theta \bar{\varphi})_{1}(t)\right|+\mid(\Theta \varphi)_{2}(t) & -(\Theta \bar{\varphi})_{2}(t) \mid \\
& \leq K \int_{0}^{t}\left(\left|\varphi_{1}(s)-\bar{\varphi}_{1}(s)\right|+\left|\varphi_{2}(s)-\bar{\varphi}_{2}(s)\right|\right) d s .
\end{aligned}
$$

On the space $\mathcal{X}_{T}$ we define a new metric by the formula

$$
\rho(\varphi, \bar{\varphi})=\sup _{t \in[0, T]} e^{-\gamma t}\left(\left|\varphi_{1}(t)-\bar{\varphi}_{1}(t)\right|+\left|\varphi_{2}(t)-\bar{\varphi}_{2}(t)\right|\right),
$$

where $\gamma$ is a positive constant (see [3]). Clearly

$$
\begin{aligned}
e^{-\gamma T} \sup _{t \in[0, T]}\left(\mid \varphi_{1}(t)\right. & \left.-\bar{\varphi}_{1}(t)|+| \varphi_{2}(t)-\bar{\varphi}_{2}(t) \mid\right) \\
\leq \rho(\varphi, \bar{\varphi}) & \leq \sup _{t \in[0, T]}\left(\left|\varphi_{1}(t)-\bar{\varphi}_{1}(t)\right|+\left|\varphi_{2}(t)-\bar{\varphi}_{2}(t)\right|\right) .
\end{aligned}
$$

Thus $\rho$ is equivalent to the usual metric defined by 3.8$)$ in $C\left([0, T], \mathbb{R}^{2}\right)$, and $\left(\mathcal{X}_{T}, \rho\right)$ is a complete metric space. We have

$$
\begin{aligned}
e^{-\gamma t}\left(\left|(\Theta \varphi)_{1}(t)-(\Theta \bar{\varphi})_{1}(t)\right|+\mid\right. & \left.(\Theta \varphi)_{2}(t)-(\Theta \bar{\varphi})_{2}(t) \mid\right) \\
& \leq K e^{-\gamma t} \int_{0}^{t} e^{\gamma s} d s \cdot \rho(\varphi, \bar{\varphi})=\frac{K}{\gamma} \rho(\varphi, \bar{\varphi}) .
\end{aligned}
$$

Since the right-hand side does not depend on $t$, we observe that

$$
\begin{aligned}
\rho(\Theta \varphi, \Theta \bar{\varphi}) & =\sup _{t \in[0, T]} e^{-\gamma t}\left(\left|(\Theta \varphi)_{1}(t)-(\Theta \bar{\varphi})_{1}(t)\right|+\left|(\Theta \varphi)_{2}(t)-(\Theta \bar{\varphi})_{2}(t)\right|\right) \\
& \leq \frac{K}{\gamma} \rho(\varphi, \bar{\varphi}) .
\end{aligned}
$$

Choose $\gamma$ such that $K / \gamma<1$. The assertion of our theorem follows from the Banach fixed point theorem.

COROLlary 4.2. The system of differential equations (2.1)-(2.4) with the initial conditions (3.1) has exactly one solution on the set

$$
x \geq 0, \quad t \in[0, \infty) .
$$

5. Reduced model. A natural problem is to contrast the above model and the classical Lotka-Volterra model. To do that we will consider a reduced 
model. Define $z_{i}$ to be the global numbers of individuals, i.e.

$$
z_{i}(t)=\int_{0}^{\infty} u_{i}(t, x) d x, \quad i=1,2 .
$$

Clearly, we assume that $z_{1}, z_{2}>0$.

Assume that the $u_{i}$ are differentiable and

$$
\lim _{x \rightarrow \infty} u_{i}(t, x)=0 .
$$

Since

$$
\int_{0}^{\infty} \frac{\partial u_{i}}{\partial x} d x=-u_{i}(t, 0)
$$

we obtain

$$
\begin{aligned}
& z_{1}^{\prime}(t)=k \int_{0}^{\infty} \int_{0}^{\infty} \alpha(x, y) u_{1}(t, y) u_{2}(t, x) d x d y-\int_{0}^{\infty} \lambda(x) u_{1}(t, x) d x, \\
& z_{2}^{\prime}(t)=\int_{0}^{\infty} \beta(x) u_{2}(t, x) d x-\int_{0}^{\infty} \int_{0}^{\infty} \alpha(x, y) u_{1}(t, y) u_{2}(t, x) d x d y .
\end{aligned}
$$

To obtain the classical Lotka-Volterra model we must assume that

$$
\alpha(x, y)=\bar{\alpha}, \quad \lambda(x)=\bar{\lambda}, \quad \beta(x)=\bar{\beta} .
$$

In this situation the problem (2.1)- 2.4 has the form

$$
\left\{\begin{array}{l}
\frac{\partial u_{1}}{\partial t}+\frac{\partial u_{1}}{\partial x}=-\bar{\lambda} u_{1}(t, x) \\
\frac{\partial u_{2}}{\partial t}+\frac{\partial u_{2}}{\partial x}=-\bar{\alpha} z_{1}(t) \cdot u_{2}(t, x) \\
u_{2}(t, 0)=\bar{\beta} z_{2}(t) \\
u_{1}(t, 0)=k \bar{\alpha} z_{1}(t) z_{2}(t) \\
z_{1}(t)=\int_{0}^{\infty} u_{1}(t, x) d x \\
z_{2}(t)=\int_{0}^{\infty} u_{2}(t, x) d x
\end{array}\right.
$$

Integrating the first two equations with respect to $x$ and assuming that $\lim _{x \rightarrow \infty} u_{i}(t, x)=0$ for $i=1,2$ we obtain the system

$$
\left\{\begin{array}{l}
z_{1}^{\prime}(t)-u_{1}(t, 0)=-\bar{\lambda} z_{1}(t) \\
z_{2}^{\prime}(t)-u_{2}(t, 0)=-\bar{\alpha} z_{1}(t) z_{2}(t),
\end{array}\right.
$$

and consequently

$$
\left\{\begin{array}{l}
z_{1}^{\prime}(t)=k \bar{\alpha} z_{1}(t) z_{2}(t)-\bar{\lambda} z_{1}(t) \\
z_{2}^{\prime}(t)=\bar{\beta} z_{2}(t)-\bar{\alpha} z_{1}(t) z_{2}(t) .
\end{array}\right.
$$


The last system is the classical Lotka-Volterra system. From [13] it follows that for all initial values $\left(z_{1}, z_{2}\right)$ the solution of this system is a pair of periodic functions with the same period.

Let now $\left(v_{1}, v_{2}\right):[0, \infty) \rightarrow \mathbb{R}^{2}$ satisfy the following conditions:

- $v_{1}, v_{2} \in L^{1}([0, \infty))$;

- $v_{i}(x) \geq 0$ for $i=1,2$ and for every $x \geq 0$;

- $\lim _{x \rightarrow \infty} v_{i}(x)=0$ for $i=1,2$;

- $\int_{0}^{\infty} v_{i}(x) d x \geq 0$ for $i=1,2$.

Let $z=\left(z_{1}, z_{2}\right): \mathbb{R}_{+} \rightarrow \mathbb{R}^{2}$ be the solution of (5.4) with the initial condition

$$
z_{i}(0)=\int_{0}^{\infty} v_{i}(x) d x \quad \text { for } i=1,2 .
$$

Let

$$
\begin{aligned}
& u_{1}(t, x)= \begin{cases}k \bar{\alpha} z_{1}(t-x) z_{2}(t-x) e^{-\bar{\lambda} x} & \text { for } x \leq t, \\
v_{1}(x-t) e^{-\bar{\lambda} t} & \text { for } x>t,\end{cases} \\
& u_{2}(t, x)= \begin{cases}\bar{\beta} z_{2}(t-x) e^{-\bar{\alpha} \int_{t-x}^{t} z_{1}(s) d s} & \text { for } x \leq t, \\
v_{2}(x-t) e^{-\bar{\alpha} \int_{0}^{t} z_{1}(s) d s} & \text { for } x>t .\end{cases}
\end{aligned}
$$

LEMma 5.1. Under the assumption (5.2) the function defined by the formulas (5.5), (5.6) is a solution to (2.1) -(2.4) in the sense of Definition 3.1.

Proof. It is sufficient to show that

$$
z_{i}(t)=\int_{0}^{\infty} u_{i}(t, x) d x
$$

For $i=1$ we have

$$
\int_{0}^{\infty} u_{1}(t, x) d x=k \bar{\alpha} \int_{0}^{t} z_{1}(t-x) z_{2}(t-x) e^{-\bar{\lambda} x} d x+\int_{t}^{\infty} v_{1}(x-t) e^{-\bar{\lambda} t} d x .
$$

The second summand is clearly equal to $z_{1}(0) e^{-\bar{\lambda} t}$. To calculate the first, we make a simple change of variables

$$
\begin{aligned}
\int_{0}^{t} z_{1}(t-x) z_{2}(t-x) e^{-\bar{\lambda} x} d x & =\int_{0}^{t} z_{1}(x) z_{2}(x) e^{-\bar{\lambda}(t-x)} d x \\
& =e^{-\bar{\lambda} t} \int_{0}^{t} z_{1}(x) z_{2}(x) e^{\bar{\lambda} x} d x .
\end{aligned}
$$

From (5.4) it follows that

$$
k \bar{\alpha} z_{1}(t) z_{2}(t)=z_{1}^{\prime}(t)+\bar{\lambda} z_{1}(t) .
$$


Then

$$
\begin{aligned}
k \bar{\alpha} \int_{0}^{t} z_{1}(x) z_{2}(x) e^{\bar{\lambda} x} d x & =\int_{0}^{t}\left(z_{1}^{\prime}(x)+\bar{\lambda} z_{1}(x)\right) e^{\bar{\lambda} x} d x \\
& =\int_{0}^{t} \frac{d}{d x}\left(z_{1}(x) e^{\bar{\lambda} x}\right) d x=z_{1}(t) e^{\bar{\lambda} t}-z_{1}(0)
\end{aligned}
$$

and so

$$
\int_{0}^{\infty} u_{1}(t, x) d x=e^{-\bar{\lambda} t}\left(z_{1}(t) e^{\bar{\lambda} t}-z_{1}(0)\right)+e^{-\bar{\lambda} t} z_{1}(0)=z_{1}(t) .
$$

To prove the equality for $i=2$ we define

$$
Z_{1}(t)=\int_{0}^{t} z_{1}(s) d s
$$

Then

$$
\int_{0}^{\infty} u_{2}(t, x) d x=\bar{\beta} \int_{0}^{t} z_{2}(t-x) e^{-\bar{\alpha}\left(Z_{1}(t)-Z_{1}(t-x)\right)} d x+\int_{t}^{\infty} v_{2}(x-t) e^{-\bar{\alpha} Z_{1}(t)} d x .
$$

Analogously, for $i=1$ the second summand is equal to

$$
e^{-\bar{\alpha} Z_{1}(t)} z_{2}(0)
$$

and the first to

$$
\bar{\beta} e^{-\bar{\alpha} Z_{1}(t)} \int_{0}^{t} z_{2}(x) e^{\bar{\alpha} Z_{1}(x)} d x .
$$

From (5.4) it follows that

$$
\bar{\beta} z_{2}(t)=z_{2}^{\prime}(t)+\bar{\alpha} z_{1}(t) z_{2}(t) .
$$

Consequently,

$$
\bar{\beta} z_{2}(t) e^{\bar{\alpha} Z_{1}(t)}=\left(z_{2}(t) e^{\bar{\alpha} Z_{1}(t)}\right)^{\prime} .
$$

From the last equality it follows that

$$
\int_{0}^{\infty} u_{2}(t, x) d x=e^{-\bar{\alpha} Z_{1}(t)}\left(\int_{0}^{t} \frac{d}{d x}\left(z_{2}(x) e^{\bar{\alpha} Z_{1}(x)}\right) d x+z_{2}(0)\right)=z_{2}(t) .
$$

This completes the proof.

Consider now the system (5.4). It is obvious that it has a periodic solution on the whole set $\mathbb{R}_{+}$. Let $\left(z_{1}, z_{2}\right): \mathbb{R} \rightarrow \mathbb{R}^{2}$. Define $\left(\widetilde{v}_{1}, \widetilde{v}_{2}\right)$ by

$$
\begin{aligned}
& \widetilde{v}_{1}(x)=k \bar{\alpha} z_{1}(-x) z_{2}(-x) e^{-\bar{\lambda} x}, \\
& \widetilde{v}_{2}(x)=\bar{\beta} z_{2}(-x) e^{-\bar{\alpha} \int_{-x}^{0} z_{1}(s) d s} .
\end{aligned}
$$


We show that $\widetilde{v}_{1}, \widetilde{v}_{2} \in L^{1}([0, \infty))$. For $T>0$ we have

$$
\begin{aligned}
\int_{0}^{T} \widetilde{v}_{1}(x) d x & =\int_{0}^{T} k \bar{\alpha} z_{1}(-x) z_{2}(-x) e^{-\bar{\lambda} x} d x=\int_{-T}^{0} k \bar{\alpha} z_{1}(x) z_{2}(x) e^{\bar{\lambda} x} d x \\
& =\int_{-T}^{0} \frac{d}{d x}\left(z_{1}(x) e^{\bar{\lambda} x}\right) d x=z_{1}(0)-e^{-T} z_{1}(-T) .
\end{aligned}
$$

Since $z_{1}$, being continuous and periodic, is bounded, we have

$$
\lim _{T \rightarrow \infty} z_{1}(-T) e^{-\lambda T}=0,
$$

which implies that

$$
\int_{0}^{\infty} \widetilde{v}_{1}(x) d x=z_{1}(0)
$$

Analogously

$$
\begin{aligned}
\int_{0}^{T} \widetilde{v}_{2}(x) d x & =\int_{0}^{T} \bar{\beta} z_{2}(-x) e^{-\bar{\alpha} \int_{-x}^{0} z_{1}(s) d s} d x \\
& =\int_{-T}^{0} \bar{\beta} z_{2}(x) e^{-\bar{\alpha} \int_{x}^{0} z_{1}(s) d s} d x=\int_{-T}^{0} \bar{\beta} z_{2}(x) e^{\bar{\alpha} Z_{1}(x)} d x
\end{aligned}
$$

where for $x<0$,

$$
Z_{1}(x)=-\int_{x}^{0} z_{1}(s) d s
$$

Then an argument analogous to the proof of Lemma 5.1 yields

$$
\int_{0}^{T} \widetilde{v}_{2}(x) d x=\int_{-T}^{0} \frac{d}{d x}\left(z_{2}(x) e^{\bar{\alpha} Z_{1}(x)}\right) d x=z_{2}(0)-z_{2}(-T) e^{\bar{\alpha} Z_{1}(-T)} .
$$

To complete the proof it is sufficient to notice that since $z_{1}$ is periodic and positive, we have

$$
\lim _{T \rightarrow \infty} e^{\bar{\alpha} Z_{1}(-T)}=0,
$$

and so

$$
\int_{0}^{\infty} \widetilde{v}_{2}(x) d x=z_{2}(0)
$$

Now, we shall prove the following theorem.

THEOREM 5.2. Let $\left(\widetilde{u}_{1}, \widetilde{u}_{2}\right)$ be the solution to problem (5.3) with the initial conditions

$$
\widetilde{u}_{i}(0, x)=\widetilde{v}_{i}(x) \quad \text { for } i=1,2 .
$$

Then the functions $\widetilde{u}_{1}, \widetilde{u}_{2}$ are periodic in $t$. 
Proof. First we recall the formula (5.5). For $x \leq t$,

$$
\widetilde{u}_{1}(t, x)=k \bar{\alpha} z_{1}(t-x) z_{2}(t-x) e^{-\bar{\lambda} x}
$$

and for $x>t$,

$$
\widetilde{u}_{1}(t, x)=\widetilde{v}_{1}(x-t) e^{-\bar{\lambda} t}=k \bar{\alpha} z_{1}(t-x) z_{2}(t-x) e^{-\bar{\lambda}(x-t)} e^{-\bar{\lambda} t} .
$$

Then for every $x \in \mathbb{R}_{+}$,

$$
\widetilde{u}_{1}(t, x)=k \bar{\alpha} z_{1}(t-x) z_{2}(t-x) e^{-\bar{\lambda} x}
$$

and the periodicity of $z_{1}$ and $z_{2}$ implies the periodicity of $\widetilde{u}_{1}$. The proof of the periodicity of $\widetilde{u}_{2}$ is analogous.

Let $V$ be the space of all non-negative functions $\left(v_{1}, v_{2}\right): \mathbb{R}_{+} \rightarrow \mathbb{R}^{2}$ such that

- $v_{i} \in L^{1}\left(\mathbb{R}_{+}\right)$for $i=1,2$;

- $v_{i}$ is continuous for $i=1,2$;

- $\lim _{x \rightarrow \infty} v_{i}(x)=0$ for $i=1,2$;

- $v_{1}(0)=k \bar{\alpha} \int_{0}^{\infty} \int_{0}^{\infty} v_{2}(x) v_{1}(y) d x d y$;

- $v_{2}(0)=\bar{\beta} \int_{0}^{\infty} v_{2}(x) d x$.

Let $T_{t}: V \rightarrow V$ be defined by the formula

$$
T_{t} v(x)=u(t, x),
$$

where $u=\left(u_{1}, u_{2}\right)$ is the solution of problem (5.3) with the initial conditions

$$
u_{i}(0, x)=v_{i}(x) \quad \text { for } i=1,2 .
$$

It is obvious that $\left\{T_{t}\right\}_{t \geq 0}$ is a semidynamical system on $V$. From Theorem 5.2 it follows that this system has periodic trajectories. Moreover, for any $z_{1}, z_{2}>0$ there exists a periodic point $\left(v_{1}, v_{2}\right)$ of the system $\left\{T_{t}\right\}$ such that $\left\|v_{i}\right\|_{L^{1}}=z_{i}(0)$ for $i=1,2$. Now, we shall prove that this system is asymptotically periodic, i.e. the set of periodic trajectories is an attractor of the system.

Theorem 5.3. Let $\left(v_{1}, v_{2}\right) \in V$ and let $z_{i}=\int_{0}^{\infty} v_{i}(x) d x$ for $i=1,2$. Let $\widetilde{v}=\left(\widetilde{v}_{1}, \widetilde{v}_{2}\right)$ be defined by (5.8), (5.9). Then

$$
\lim _{t \rightarrow \infty}\left\|T_{t} v_{i}-T_{t} \widetilde{v}_{i}\right\|=0 \quad \text { for } i=1,2 .
$$

Proof. From (5.5) and (5.6) it follows that

$$
\begin{aligned}
& T_{t} v_{1}(x)= \begin{cases}k \bar{\alpha} z_{1}(t-x) z_{2}(t-x) e^{-\bar{\lambda} x} & \text { for } x \leq t, \\
v_{1}(x-t) e^{-\bar{\lambda} t} & \text { for } x>t,\end{cases} \\
& T_{t} v_{2}(x)= \begin{cases}\bar{\beta} z_{2}(t-x) e^{-\bar{\alpha} \int_{t-x}^{t} z_{1}(s) d s} & \text { for } x \leq t, \\
v_{2}(x-t) e^{-\bar{\alpha} \int_{0}^{t} z_{1}(s) d s} & \text { for } x>t .\end{cases}
\end{aligned}
$$


Moreover for $x>t$ we have

$$
T_{t} \widetilde{v}_{1}\left(x=\widetilde{v}_{1}(x-t) e^{-\bar{\lambda} t}, \quad T_{t} \widetilde{v}_{2}(x)=\widetilde{v}_{2}(x-t) e^{-\bar{\alpha} \int_{0}^{t} z_{1}(s) d s},\right.
$$

while for $x \leq t$ we have $T_{t} \widetilde{v}_{1}(x)=T_{t} v_{1}(x)$ and $T_{t} \widetilde{v}_{2}(x)=T_{t} v_{2}(x)$. It follows that for every $t>0$ and $i=1,2$,

$$
\left\|T_{t} v_{i}-T_{t} \widetilde{v}_{i}\right\|=\int_{t}^{\infty}\left|T_{t} v_{i}(x)-T_{t} \widetilde{v}_{i}(x)\right| d x \leq \int_{t}^{\infty}\left|T_{t} v_{i}(x)\right| d x+\int_{t}^{\infty}\left|T_{t} \widetilde{v}_{i}(x)\right| d x .
$$

It is obvious that

$$
\begin{gathered}
\int_{t}^{\infty}\left|T_{t} v_{1}(x)\right| d x \leq e^{-\bar{\lambda} t}\left\|v_{1}\right\|_{L^{1}}, \quad \int_{t}^{\infty}\left|T_{t} \widetilde{v}_{1}(x)\right| d x \leq e^{-\bar{\lambda} t}\left\|\widetilde{v}_{1}\right\|_{L^{1}}, \\
\int_{t}^{\infty}\left|T_{t} v_{2}(x)\right| d x \leq e^{-\bar{\alpha} \int_{0}^{t} z_{1}(s) d s}\left\|v_{2}\right\|_{L^{1}}, \int_{t}^{\infty}\left|T_{t} \widetilde{v}_{2}(x)\right| d x \leq e^{-\bar{\alpha} \int_{0}^{t} z_{1}(s) d s}\left\|\widetilde{v}_{2}\right\|_{L^{1}} .
\end{gathered}
$$

This completes the proof.

6. Conclusions. The model presented in this paper is significantly more general than the classical Lotka-Volterra model. In particular, in our model the existence of a positive stationary solution depends on the reproductive capability of preys. This approach is obvious from the biological point of view. In the last section we consider the reduced model, i.e we take the age structure into consideration, but it has no influence on the development of the population. It can be seen that in this model the age structure of both predators and preys stabilizes.

Acknowledgements. The second author acknowledges the support from Białystok University of Technology (grant No. S/WI/2/2011).

\section{References}

[1] N. C. Apreutesei, Necessary optimality conditions for predator-prey system with a hunter population, Opuscula Math. 30 (2010), 389-397.

[2] - Necessary optimality conditions for a Lotka-Volterra three species system, Math. Modelling Natural Phenomena 1 (2006), 123-135.

[3] A. Bielecki, Une remarque sur la méthode de Banach-Caccioppoli-Tikhonov dans la théorie des équations différentielles ordinaires, Bull. Acad. Polon. Sci. 4 (1956), 261-268.

[4] L. M. Cai, X. Z. Li, X. Y. Song and J. Y. Yu, Permanence and stability of an age-structured prey-predator system with delays, Discrete Dynam. Nat. Soc. 2007, art. ID 54861, 15 pp.

[5] J. M. Cushing and M. Saleem, A predator prey model with age structure, J. Math. Biol. 14 (1982), 231-250; Erratum, ibid. 16 (1982/83), 305.

[6] A. L. Dawidowicz and A. Poskrobko, Age-dependent single-species population dynamics with delayed argument, Math. Methods Appl. Sci. 33 (2010), 1122-1135. 
[7] A. L. Dawidowicz and J. L. Zalasiński, On the system of von Foerster equations with nonlinear boundary conditions, in: Proc. 4th National Conference on Application of Mathematics in Biology and Medicine (Zwierzyniec, 1998), 24-28.

[8] M. Delgado and A. Suárez, Age-dependent diffusive Lotka-Volterra type system, Math. Comput. Modelling 45 (2007), 668-680.

[9] B. Dubey, A prey-predator model with a reserved area, Nonlinear Anal. Modeling Control 12 (2007), 479-494.

[10] U. Foryś, Multi-dimensional Lotka-Volterra systems for carcinogenesis mutations, Math. Methods Appl. Sci. 32 (2009), 2287-2308.

[11] M. E. Gurtin and R. C. MacCamy, Non-linear age-dependent population dynamics, Arch. Ration. Mech. Anal. 54 (1974), 281-300.

[12] A. G. McKendrick, Application of mathematics to medical problems, Proc. Edinburgh Math. Soc. 44 (1926), 98-130.

[13] V. Volterra, Variations and fluctuations of the number of individuals in animal species living together, in: Animal Ecology, McGraw-Hill, 1931, 409-448.

[14] J. von Foerster, Some remarks on changing populations, in: The Kinetics of Cell Proliferation, Grune \& Stratton, New York, 1959, 382-407.

[15] W. X. Xu, T. L. Zhang and Z. B. Xu, Existence of positive periodic solutions of a prey-predator system with several delays, Acta Math. Sci. Ser. A Chinese Ed. 28 (2008), 39-45.

Antoni Leon Dawidowicz

Institute of Mathematics

Jagiellonian University

30-348 Kraków, Poland

E-mail: Antoni.Leon.Dawidowicz@im.uj.edu.pl

Jerzy Leszek Zalasiński

Tarnów Regional Development Agency SA

33-100 Tarnów, Poland

E-mail: Jerzy.Zalasinski@gmail.com
Anna Poskrobko

Faculty of Computer Science Białystok University of Technology 15-351 Białystok, Poland E-mail: a.poskrobko@pb.edu.pl

Received on 15.4.2010;

revised version on 22.4.2011 
